\title{
The relationship of myocardial contraction and electrical excitation-the correlation between scintigraphic phase image analysis and electrophysiologic mapping
}

\author{
L. Munoz del Romeral, MD, PhD, ${ }^{\mathrm{a}}$ C. Stillson, BS, ${ }^{\mathrm{b}} \mathrm{M}$. Lesh, $\mathrm{MD},{ }^{\mathrm{c}}$ M. Dae, MD, ${ }^{\mathrm{b}}$ \\ and Elias Botvinick, $M D^{\mathrm{b}}$
}

Background. Phase imaging derived from equilibrium radionuclide angiography presents the ventricular contraction sequence. It has been widely but only indirectly correlated with the sequence of electrical myocardial activation.

Objectives. We sought to determine the specific relationship between the sequence of phase progression and the sequence of myocardial activation, contraction and conduction, in order to document a noninvasive method that could monitor both.

Methods. In 7 normal and 9 infarcted dogs, the sequence of phase angle was correlated with the epicardial activation map in 126 episodes of sinus rhythm and pacing from three ventricular sites.

Results. In each episode, the site of earliest phase angle was identical to the focus of initial epicardial activation. Similarly, the serial contraction pattern by phase image analysis matched the electrical epicardial activation sequence completely or demonstrated good agreement in approximately $85 \%$ of pacing episodes, without differences between normal or infarct groups.

Conclusions. A noninvasive method to accurately determine the sequence of contraction may serve as a surrogate for the associated electrical activation sequence or be applied to identify their differences. (J Nucl Cardiol 2009;16:792-800.)

Key Words: Contraction • excitation $\cdot$ equilibrium radionuclide angiograms $\cdot$ phase image analysis

The manuscript is from UCSF not from Alta Bates or Evera. The latter is where the respective people currently work.

From the Division of Heart Failure and Pulmonary Hypertension, ${ }^{\mathrm{a}}$ Alta Bates Hospital, Berkeley, CA; Departments of Medicine, Cardiovascular Division, and Radiology, Nuclear Medicine Section, and the Cardiovascular Research Institute, ${ }^{b}$ University of California San Francisco, San Francisco, CA; Evera Medical, Inc., ${ }^{\mathrm{c}}$ Foster City, CA.

The authors had full access to the data and take full responsibility for its integrity. All authors have read and agree to the article as written.

Received for publication Mar 16, 2009; final revision accepted Jun 11, 2009.

Reprint requests: Elias Botvinick, MD, Departments of Medicine, Cardiovascular Division, and Radiology, Nuclear Medicine Section, and the Cardiovascular Research Institute, University of California San Francisco, 505 Parnassus Avenue, Box 0214, San Francisco, CA 94143; botvinicke@medicine.ucsf.edu.

1071-3581/ $\$ 34.00$

Copyright (C) 2009 The Author(s). This article is published with open access at Springerlink.com.

doi:10.1007/s12350-009-9114-9

\section{INTRODUCTION}

Phase image analysis, a functional image derived from the equilibrium radionuclide angiogram (ERNA), graphically displays the regional phase angle, a parameter which relates to regional ventricular volume change. In this way, the phase image presents the ventricular contraction sequence. In the presence of electromechanical association, this contraction pattern has been found to correlate with the overall pattern of myocardial electrical activation and conduction. ${ }^{1,2}$ The site of earliest wall motion and the sequence of wall motion on phase imaging has well localized the earliest site and the pattern of electrical excitation on ECG and electrophysiologic study in pre-excitation and a variety of conduction abnormalities. $^{3-9}$ The pattern of phase angle progression has also been demonstrated to roughly parallel the conduction sequence with a variety of conduction abnormalities 
and ectopic rhythms. ${ }^{5,7,9,10}$ A recent application analyzes the sequence of wall motion to determine indices of ventricular synchrony. ${ }^{11}$

However, the progression of phase angle, and the sequence of related contraction, has never been carefully correlated with detailed epicardial mapping. Documentation of the correlation between the phase image pattern of sequential contraction with electrical conduction in subjects in normal sinus rhythm (NSR) and ventricular pacing with normal and scarred ventricles would establish the phase imaging method as a noninvasive image correlate of both function and conduction and would also serve as a basis for understanding, on a gross scale, the relationship between contraction and conduction. The phase method could potentially benefit the understanding and treatment of myocardial dysfunction and heart failure.

Systolic ventricular function is closely related to the pattern and efficiency of myocardial contraction. These contribute to myocardial contraction synchrony and are greatly influenced by the activation pattern. A noninvasive imaging method to accurately display the sequence of myocardial contraction could be a surrogate for the pattern of myocardial activation and conduction and provide a measure of synchrony. ${ }^{11}$ This might be especially valuable in the assessment of clinical conditions, as advanced systolic heart failure, where symptoms and even survival depend on myocardial synchrony and the relationship between conduction and related contraction. Dual chamber ventricular pacemakers have been shown to improve ventricular function and symptoms in patients with advanced heart failure. ${ }^{12-14}$ This likely relates to the altered pattern of conduction and related contraction through the myocardium admixed with scar. ${ }^{15}$

In an animal pacing model applied in healthy dogs and dogs with a small myocardial infarction, we correlated the site of earliest ventricular contraction and the serial ventricular contraction pattern identified by ERNA phase angle progression, with the site of earliest ventricular electrical activation and the serial regional activation pattern defined by the epicardial activation map (EAM).

\section{METHODS}

The following protocol was applied in 16 healthy mongrel dogs weighing 24-35 kg, including 7 normal dogs and 9 dogs which were instrumented one month after producing a limited distal anteroseptal myocardial infarction. Small infarctions were sought to avoid the effects of large dense scar and encourage animal survival. All experiments were performed in accordance with institutional and NIH guidelines.

Infarction was produced through a left subcostal thoracotomy using a Harris two-stage open-chest coronary artery ligation procedure. ${ }^{16}$ The ligation was performed beyond the first diagonal vessel at the mid-left anterior descending coronary artery level. To obtain a detectable transmural infarction, ligations of all visible epicardial collateral vessels were performed.

Post-absorptive animals were pre-medicated with morphine sulfate, and anesthetized with I.V. alpha chloralose. They were then intubated, oxygenated and mechanically ventilated. Hydration was maintained with infusion of normal saline and body temperature supported with a thermal K-pad blanket. The surface electrocardiogram (ECG) was constantly monitored. A right femoral artery cannula was placed to continuously monitor blood pressure in the abdominal aorta.

\section{Surgical Procedure}

A median sternotomy exposed the heart, pacing electrodes and a monitoring sock array were positioned, and the heart was placed in a pericardial cradle. When pacing and recording abilities were confirmed, the proximal terminals of the electrodes were exteriorized and the thoracotomy closed. The animals were later paced in multiple sessions, from three positions: high septum (HS), right ventricular free wall (RVW) and LV apex (LVa).

With baseline NSR and with each pacing episode, ERNA was acquired in anterior and left anterior oblique views to determine the site of earliest left (LV) and right ventricular (RV) phase angle $(\varnothing)$ and the sequence of $\varnothing$ progression. An epicardial isochronous map was simultaneously generated from the sock array. Abdominal aortic pressure was recorded continuously to assure hemodynamic stability. After the experiment, the animals were euthanized by barbiturate overdose.

\section{Pacing Protocol}

Bipolar epicardial pacing was performed in random order through three silver wire electrodes sutured to the HS, RVW, or LVa, at a $300 \mathrm{~ms}$ cycle length and at twice the stimulation threshold level in order to capture the ventricles with each pacing site. Simultaneous right atrial pacing abolished the potentially confounding effect of intermittent atrial contribution to LV filling. Pacing for 10 minutes permitted complete and stable data acquisition. Ten minutes between pacing episodes achieved full recovery. A Bard 56 bipolar electrode band array or "sock" (C.R. Bard, Inc., Tewksbury, MA), with 14 columns of 4 bipoles, each with 2 electrode contacts $2 \mathrm{~mm}$ apart evenly distributed around the ventricles, recorded epicardial activation electrograms. The 56 epicardial bipolar electrograms and 8 surface ECGs were bandpass filtered at 30 to $500 \mathrm{~Hz}$ and 0.5 to $150 \mathrm{~Hz}$, respectively. Sampling rate for each signal was $1 \mathrm{kHz}$. Before closing the thoracotomy, the location of the three pacing wires was carefully noted and recorded, in relation to the bipolar electrodes and columns of the band array. The LAD was the $0^{\circ}$ polar marker applied to orient the "sock". Pacing wire position was related to the closest band array bipolar electrode in a diagram of the sock. During pacing, the electrograms and surface electrocardiograms were simultaneously recorded as 8 second samples, digitized on a BARD 64 Cardiac Mapping System v.B.05, and localized by the instrument in well validated commercial software to 38 ventricular segments with generation of iso- 


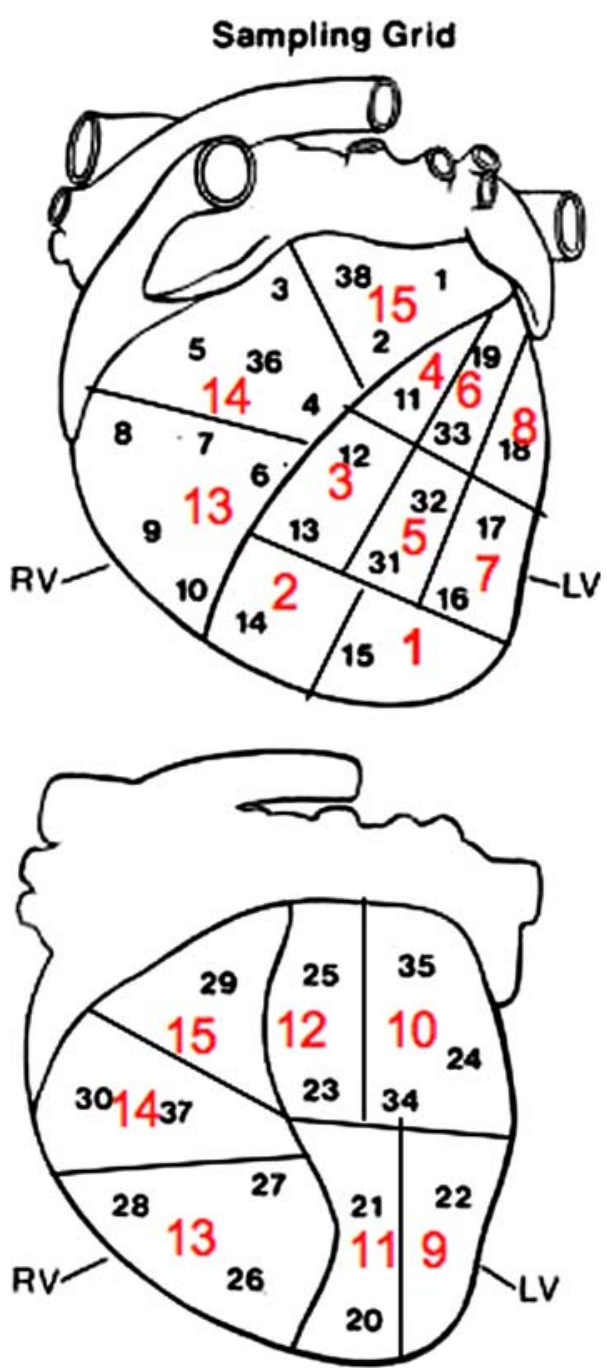

Figure 1. Thirty-eight EAM segments and 15 ventricular segments. Shown are the 38 segments mapped on EAM, correlated with the 15 ventricular segments mapped on phase analysis in anterior (above) and posterior (below) views. On the 15 segment map, segments 1 and 2 represent the left ventricular (LV) apex, 3 and 4 the mid- and basal septum, 5-8 the anterior and anterolateral LV wall, 9-12 the posterior and posterolateral LV wall and 13-15 regions of the right ventricle (RV).

chronic epicardial maps and measurement of QRS duration for each pacing episode (Figures 1 and 2). The earliest ventricular depolarization site was related to the anatomic pacing site and to the site of earliest $\varnothing$. The associated epicardial electrical activation sequence was compared to the pattern of $\varnothing$ progression for each pacing site (see subsequent sections).

\section{ERNA}

Blood pool acquisition and processing. ERNAs were acquired in 16 frames in the standard manner, on a Siemens portable scintillation camera (Siemens USA, Des Plaines, IL) equipped with a low energy all purpose collimator
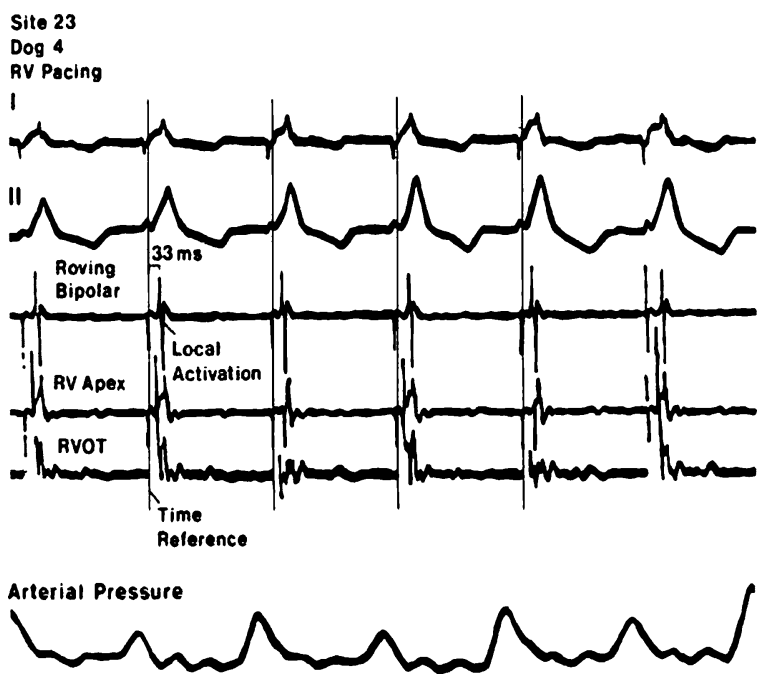

Figure 2. Electrograms. Shown are leads I and II and the electrograms generated from the RV apex and RV outflow tract (RVOT) sampled at site 23 on the EAM with RV pacing. The roving electrode sampled local activation.

and processed on a PDP 11/40 minicomputer (Digital Equipment Corp., Maynard, MA). Thirty minutes before imaging, the animals' red blood cells were labeled in vitro with $30-35 \mathrm{mCi}$ of technetium-99m. ${ }^{17}$ Images, gated to the electrocardiographic R wave, were acquired at rest, in NSR, and in all pacing modes, in the anterior and "best septal" views, the latter with $15^{\circ}$ craniocaudal angulation to minimize foreshortening of the ventricles and overlap of the atria. Image data were then digitized.

Phase analysis. Phase analysis was performed, as in prior studies, ${ }^{5}$ on images generated from a pixel-by-pixel fit of the first harmonic analysis of the blood pool data. As previously reported, ${ }^{5,10}$ blood pool images were interpolated to a $128 \times 128$ matrix format, smoothed and viewed on a high quality De Anza display using 256 gray shades and 64 colors. Black represented the earliest phase angle, near the R-wave origin, with progressively lighter shades assigned to pixels with increasing phase angle and progressively later contraction. To gain high temporal resolution we constructed a phase histogram of each ventricle, plotting phase angle on the abscissa and the number of pixels with each phase angle on the ordinate. A cursor of variable dimensions sampled the histogram and highlighted in white the related phase image pixels of any selected phase angle window width, as small as $2.8^{\circ} .^{5}$ Phase analysis permitted visualization and estimation of the sequence of ventricular contraction. The site of earliest phase angle was correlated with the site of earliest electrical activation and the pacing site, and the pattern of phase progression was correlated with the sequence of ventricular activation. Phase values were related in a relative manner to the sequence of ventricular contraction.

Study design. The animals were paced from each of the three prefixed positions. As imaging was performed, an epicardial activation map (EAM) was generated. We sought to perform and analyze two ERNAs and EAMs in each animal in NSR and at each pacing site. ERNA with subsequent phase analysis and EAM were independently evaluated by two 
different observers, each blinded to the results of the other study. The consensus was reported.

For analysis, the span of each phase histogram, plotting phase angle against the number of pixels with that phase angle, was divided into 5-7 intervals from the earliest to the latest phase angle, from the first to the last segment to contract. The regions with phase angles corresponding to the histogram interval were localized to one or more of 15 segments as conventionally mapped at EPS using the two projections available. The 38 ventricular segments of the EAM were condensed to the 15 segment map as shown in Figure 1. The anatomic region corresponding to each designated interval of the phase histogram was analyzed in the EAM and the mean electrical activation time of that area was calculated. The activation time of each segment expressed in milliseconds was ordered and compared to the contraction sequence in the related phase image. The sequence as well as the earliest and latest sites of EAM activation were correlated with those of ERNA contraction. Correlation was made based on triangulation of the phase sequence seen in the two projections, compared to the EAM sequence localized in 38 segments, condensed to parallel those 15 of the phase map.

Concordance between both methods for identification of the pacing site was achieved when the earliest contraction and the earliest activation were localized to the same anatomic segment or to an adjacent segment of the same ventricle.

Good agreement between serial contraction pattern and activation sequence was reached when there was a match between activation and contraction sequence, where the phase progression localized to the same or adjacent segments as the EAM. Partial agreement was considered present when there was a correlation of at least $50 \%$ of segments and poor when the correlation was less than $50 \%$ of the segments.

\section{STATISTICS}

The accuracy of phase analysis for identification of the site of earliest activation and sequence of activation and its agreement with the EAM in all animals and with all rhythms was determined by the chi-square test. Temporal analysis of paced segments by their sequential phase angles was correlated in each group with the temporal sequence of electrophysiologic activation by the Spearman rank order correlation. Between groups differences were compared by an unpaired $t$ test.

\section{RESULTS}

We performed 56 ERNAs with phase analysis in 7 normal dogs and 70 ERNAs in 9 infarcted animals, with concomitant EAM correlation. Studies were acquired during NSR in 32 episodes and during paced rhythm in 94 others. In infarcted dogs, postmortem exam confirmed infarction, which was always limited in extent and anterior-apical in location.

\section{Normal Dogs}

In 7 normal dogs, 14 studies were acquired during NSR, 2 in each dog, while 42 mapping studies were performed with pacing, 2 studies acquired with each of the 3 pacing sites in each animal, 6 pacing episodes per $\operatorname{dog}$ and a total of 8 mapped episodes per dog.

In normal dogs during NSR, the phase image demonstrated serial progression in parallel with the electrical activation sequence which spread rapidly through the ventricles in an organized manner from base to apex, with the LV septum first to depolarize. Phase analysis during NSR demonstrated earliest septal contraction moving to the basal RV free wall in 8 dogs and to the basal anterior LV in 6 dogs. In each case, the site of earliest phase angle on phase mapping agreed with the focus of initial epicardial electrical activation, identified by EAM. During pacing, the site of earliest phase angle was identical to the focus of initial epicardial activation on EAM, in all dogs in all 42 pacing episodes.

The serial contraction pattern by phase image analysis completely matched the electrical epicardial activation sequence with good agreement in 36 of 42 (86\%) pacing episodes, partial agreement in 4 of 42 $(10 \%)$ and poor agreement in 2 of $42(4 \%), P<.01$. In normal animals, temporal analysis of segments by their sequential phase angle correlated well with the temporal sequence of electrophysiologic activation. The related Spearman rank order correlations in these three groups was 0.94 overall.

\section{Infarcted Dogs}

In 9 infarcted dogs, 70 mapping studies were acquired including 18 studies, 2 acquired in each animal, in NSR, and 52 studies, 2 acquired in 8 animals with each of the 3 pacing sites and 4 paced episodes in one dog who could not tolerate study from the last pacing site.

During NSR, the electrical impulse again originated from sites similar to those in NSR in normal dogs and spread rapidly through the ventricles in an organized manner from base to apex, with the LV septum first to depolarize, and phase progression as in normals described above.

In relation to all 52 pacing episodes, the site of earliest ventricular contraction agreed with the focus of initial epicardial electrical activation, as defined by EAM in all dogs. The serial contraction pattern by phase image analysis matched the electrical epicardial activation sequence completely in 44 of 52 (84\%) pacing episodes, partially in 4 of $52(8 \%)$ and poorly in 4 of $52(8 \%), P<.01$ (Figures 3, 4, and 5). In 
HS Pacing - Sequential Phase Map

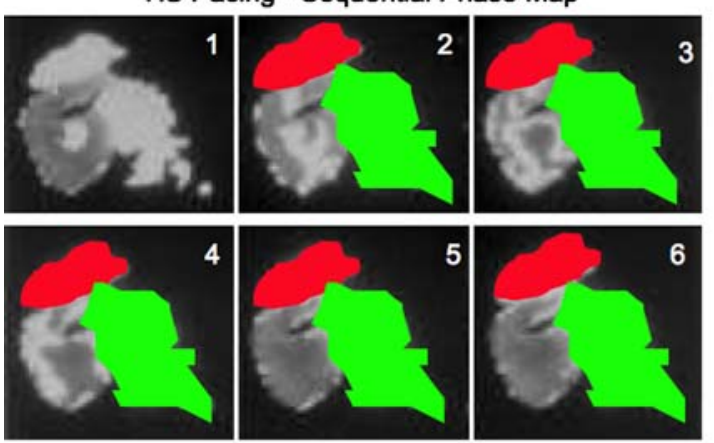

A

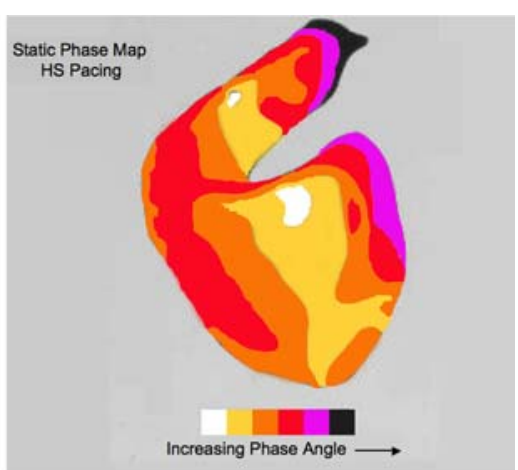

B

HS Pacing

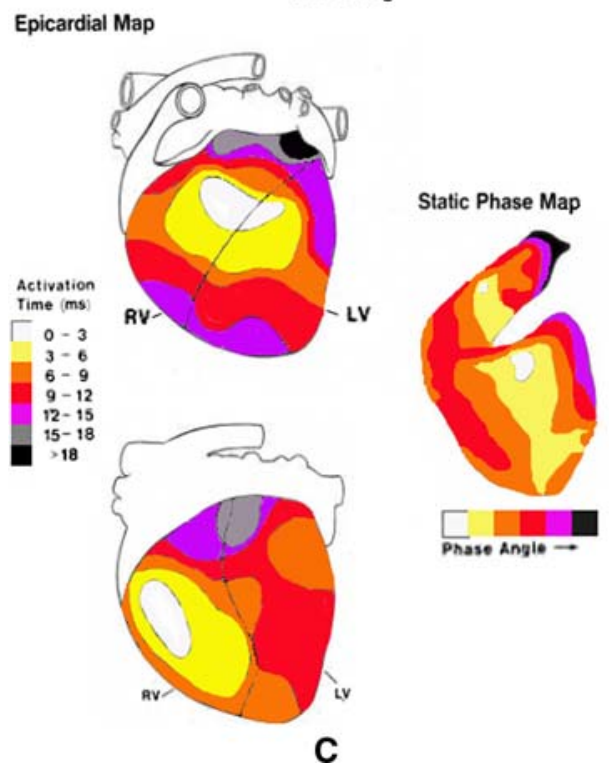

Figure 3. High septal (HS) pacing. A, Phase images. Shown in a left oblique projection are frames in a sequential phase map, from 1 through 6 , demonstrating serial progression of phase angle and related contraction, in an infarcted dog. The ventricles are in gray in the upper left image with progressive phase intervals shown in white highlight. The right atrium is masked in red and the left atrium in green. B, Phase map. Shown is the phase map drawn based on the images in A. This is a diagram characterizing the sequence of phase progression in 6 color coded intervals to match the pattern in A. Increasing phase angle and contraction delay is shown in the color code (below). The number of phase intervals illustrated in this and subsequent figures has been chosen empirically to illustrate the sequence of progression in association with contraction patterns of varying rate and complexity. C, Good phase and EAM agreement. Shown at right is the same diagram of serial phase progression, the phase map, shown in $\mathbf{B}$. At left are anterior (above) and posterior (below) views of the epicardial map presented in 7 color coded intervals. The parallel of the conduction pattern on the epicardial map with the phase map sequence is evident.

infarcted animals, temporal analysis of segments by their sequential phase angle correlated well with the temporal sequence of electrophysiologic activation (Figure 6). While the patterns of activation and contraction were somewhat altered in the presence of the small infarction, their correlation was not significantly different from that in normal dogs. The related Spearman rank order correlations in these three groups was 0.92 overall.
When the order of contraction in 286 randomly chosen LV anatomic segments on phase imaging were compared to the order of excitation on EAM, there was good agreement in 262, 92\%, $P<.001$. There was no difference between normal and infarct groups in their frequency of agreement between EAM and phase analysis in NSR and with pacing, either in the identification of the site of earliest activation/contraction or of the course of their subsequent progression. 


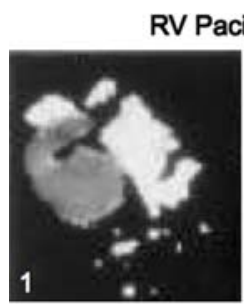

RV Pacing - Sequential Phase Map
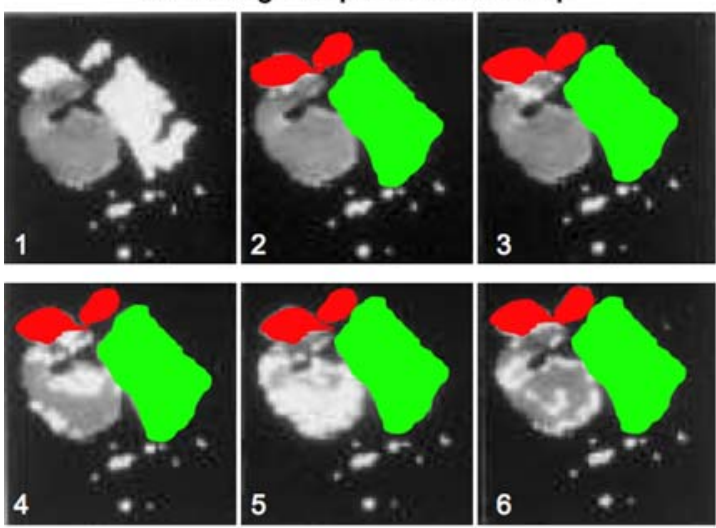

A

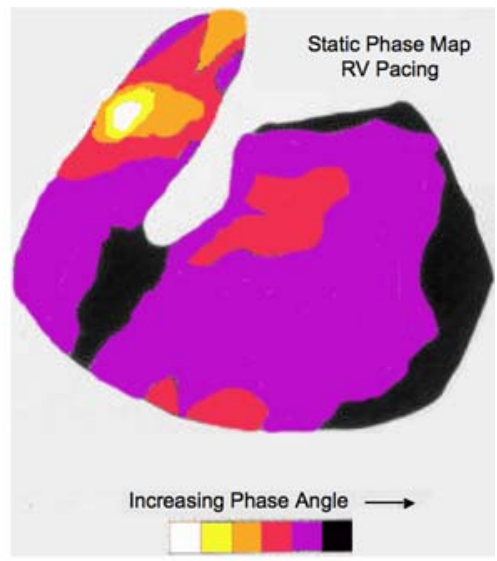

B

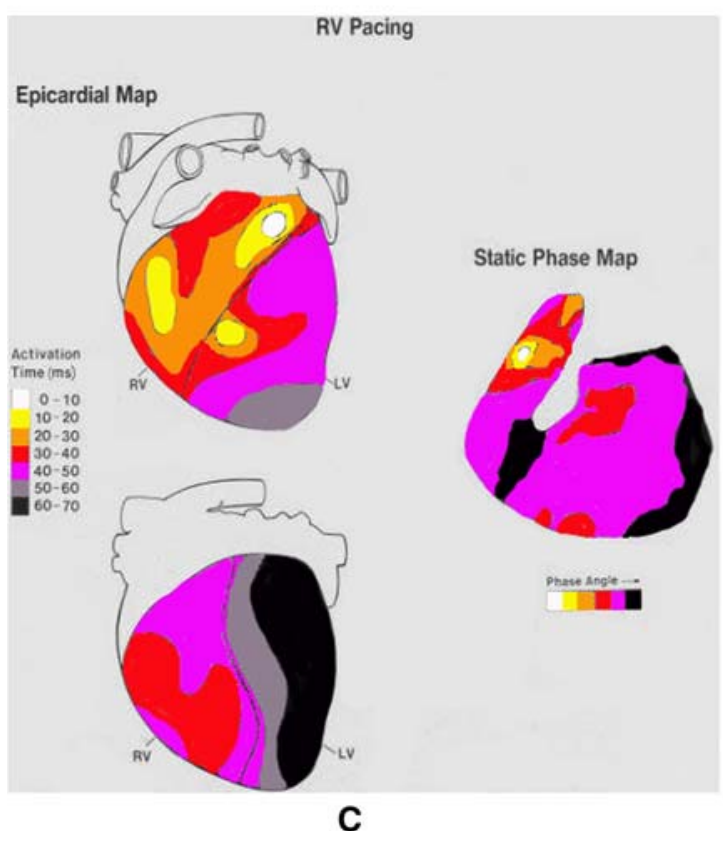

Figure 4. RV pacing. A, Phase images. Shown, as in Figure 3A, in a left oblique projection, are frames in a sequential phase map, from 1 through 6 , demonstrating serial progression of phase angle and related contraction, with RV pacing in the same dog illustrated in Figure 3. The ventricles are in gray in the upper left image with progressive phase intervals in white. The atria are again masked as in Figure 3A. B, Phase map. Shown, as in Figure 3B, is the phase map of the images in Figure 4A, a diagram characterizing the sequence of phase progression in 6 color coded intervals to match the pattern in A. C, Incomplete phase and EAM agreement. Shown at right is the same diagram of serial phase progression shown in A. At left are anterior (above) and posterior (below) views of the epicardial map presented in 7 color coded intervals. The parallels and inconsistencies with the phase map are evident.

\section{DISCUSSION}

\section{Overview}

We determined the relationship between myocardial contraction, measured by scintigraphic phase analysis, and excitation with EAM in normal dogs and those with prior infarction in NSR and with pacing. Phase analysis has not previously been so tested. The phase image findings in normal dogs were similar to those shown earlier $^{2}$ and with the established electrophysiologic pattern. $^{18}$

The small percentage of episodes with partial agreement and those few with poor agreement can be understood, given the relatively anatomic resolution of the imaging method employed. However, there was an excellent correlation between the initial site and 


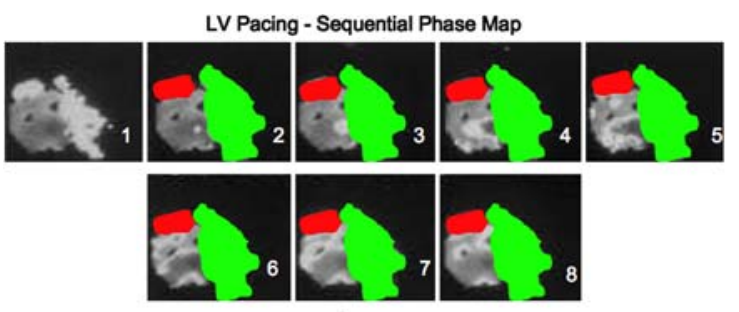

A

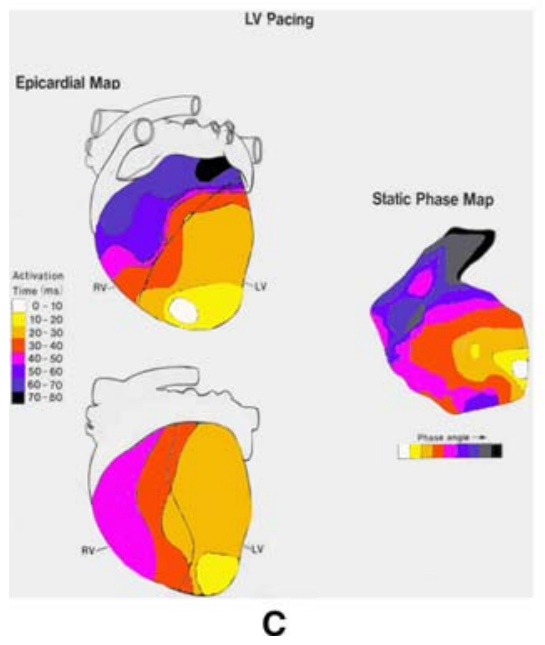

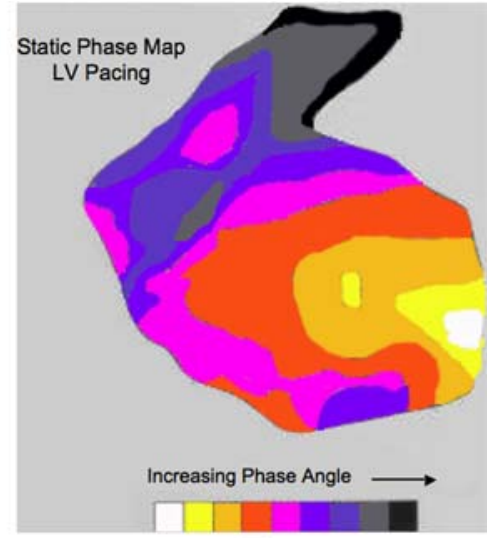

B

Figure 5. LVa pacing. A, Phase map. Shown as in Figure 3A, in a left oblique projection, are frames in a sequential phase map, from 1 through 6 , demonstrating serial progression of phase angle and related contraction, with LVa pacing in the same dog illustrated in Figures 3 and 4. The ventricles are in gray in the upper left image with progressive phase intervals sequentially highlighted in white. The atria are again masked as in Figure 3A. B, Phase map. Shown is a phase map of the images in $\mathbf{A}$, a diagram characterizing the sequence of phase progression in 9 color coded intervals to match the pattern of A. C, Good phase and EAM agreement. Shown at right is the same diagram of serial phase progression shown in A. At left are anterior (above) and posterior (below) views of the epicardial map presented in 8 color intervals. The parallel with the phase map is evident.

ventricular contraction sequence delineated on phase analysis with that on EAM in all groups studied. Such agreement indicates that the sequence and relative rate of phase angle progression corresponded to that of epicardial conduction. This is consistent with electromechanical coupling.

The high-level agreement establishes the phase method as a reliable noninvasive tool to monitor serial ventricular wall motion and related conduction. It also demonstrates the close relationship between the pattern of epicardial activation and endocardial motion in this model.

\section{Phase Analysis}

Phase image analysis presents an estimate of the sequence of regional ventricular contraction and has been extrapolated to apply to related conduction. ${ }^{2-8}$ It has localized pacemaker sites and demonstrated the relative advantages of different pacing modes. ${ }^{9}$ It has localized individual and multiple ventricular tachycardia exit sites, and mapped the subsequent pattern of ventricular contraction. ${ }^{10}$ These successful applications supported regional electromechanical association.

\section{A Noninvasive Method to Image Conduction}

Planar ERNA is a widely available and inexpensive noninvasive imaging method which permits the accurate, objective and reproducible determination of the full sequence of contraction and related electrical activation. Such assessment could be important in the evaluation of ventricular synchrony, a measure of the degree of coordination of regional ventricular wall motion, which is markedly reduced in many with systolic heart failure. Here, symptoms and even survival may depend on conduction and related contraction. 


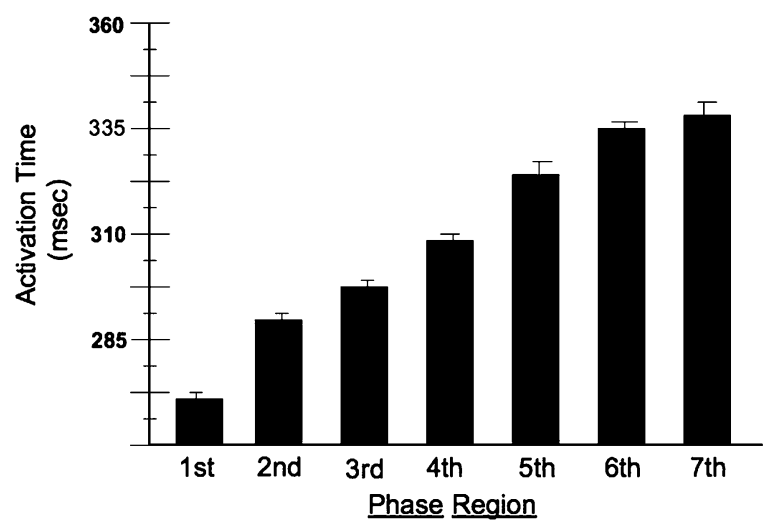

Figure 6. Phase-EAM correlation. Shown is the direct relationship between seven regions of phase progression and activation times on EAM in related segments with LVa pacing in the infarct group. A similar analysis was performed in normals and with each mapped episode with similar results. The method employed did not permit an evaluation of regional conduction or contraction rates.

The phase method has been applied to assess synchrony and predict outcome in patients with heart failure. ${ }^{19-21}$ This information is especially important in the evaluation and application of cardiac resynchronization therapy (CRT), a new treatment of advanced heart failure which seeks to restore synchrony and ventricular function by altering the sequence of conduction with biventricular pacing. ${ }^{12-15}$

New parameters of synchrony based in phase analysis promise insight into the mechanism of improvement, aid optimal pacemaker placement and present the likelihood of functional improvement with CRT. ${ }^{11,22-24}$ The ability to accurately monitor both regional contraction and the conduction sequence in a single ERNA image provides a graphic basis for subsequent studies of CRT.

\section{Study Deficiencies}

The low ERNA data density in each pixel required phase analysis, derived from a first harmonic fit of the time-activity curve. A multiharmonic fit of the curve would increase the accuracy of the curve fit. It would be required to translate phase angle into absolute timing of contraction onset. A multiharmonic fit would require greater data density than is available in a single image pixel and would need analysis of grouped pixels. Yet, a multiharmonic fit demonstrates no advantage over the first harmonic method for the evaluation of serial phase or contraction sequence. The first harmonic phase sequence correlated well with electrocardiography and electrophysiology in this and prior studies. ${ }^{2-10}$
The future development of a common template for phase and electrophysiologic evaluation will be useful and clarify any ambiguities related to localization. Figures illustrating the excitation-contraction correlation could show increasing disagreement the more refined the temporal analysis. Differences in the sampling rate between the phase image and epicardial map presented an intrinsic obstacle to exact agreement of the two. However, the data well supported the comparison of their sequence. In the figures, appropriate intervals were empirically selected to demonstrate and not obscure the relationship. The intervals displayed in phase and electrophysiologic maps were selected objectively and independently by different authors. This brought differences in the number of intervals analyzed but assured analytic objectivity.

The planar phase method is subject to errors due to structural overlap and regional contraction abnormalities. Tomographic methods may present significant localizing advantages. ${ }^{25}$ Phase image analysis would likely be more effectively applied using SPECT methods. Software for SPECT acquisition, processing and display of ERNA with phase analysis are already written for current instruments.

\section{SUMMARY}

In both normal and infarcted dogs in NSR and with pacing, a strong correlation was demonstrated between the site of initial contraction and the subsequent myocardial contraction pattern, as identified noninvasively by the sequence of ERNA derived phase angle progression, and their electrical correlates on EAM. The findings parallel those made in patients with a variety of conduction patterns and strongly support the presence of electromechanical association and the ability of the phase imaging method to map it. The findings suggest a variety of applications to identify, understand and treat conditions of ventricular dysfunction which may be adversely influenced by the pattern of conduction.

\section{Acknowledgment}

Dr. Munoz was supported by a Research Fellowship from the American Heart Association, California Affiliate.

\section{Open Access}

This article is distributed under the terms of the Creative Commons Attribution Noncommercial License which permits any noncommercial use, distribution, and reproduction in any medium, provided the original author(s) and source are credited. 


\section{References}

1. Botvinick EH, Frais MA, Shosa DW, O'Connell JW, PachecoAlvarez JA, Scheinman M, et al. An accurate means of detecting and characterizing abnormal patterns of ventricular activation by phase image analysis. Am J Cardiol 1982;50:289-98.

2. Botvinick E, Dunn R, Frais M, O'Connell W, Shosa D, Herfkens $\mathrm{R}$, et al. The phase image: Its relationship to patterns of contraction and conduction. Circulation 1982;65:551-60.

3. Frais M, Botvinick E, Shosa D, O'Connell W, Scheinman M, Hattner R, et al. Phase image characterization of ventricular contraction in left and right bundle branch block. Am J Cardiol 1982;50:95-104.

4. Dae M, Wen YM, Botvinick E, Scheinman M. EKG left anterior fascicular block reveals diverse patterns on scintigraphic phase analysis. Am Heart J 1989;117:861-9.

5. Botvinick EH, Frais M, O'Connell JW, Faulkner D, Scheinman MM, Morady F, et al. Phase image evaluation of patients with ventricular pre-excitation syndromes. J Am Coll Cardiol 1984;3:799-814.

6. Schechtmann N, Botvinick E, Dae M, Scheinman MM, O'Connell JW, Davis J, et al. The scintigraphic characteristics of ventricular pre-excitation through Mahaim fibers with the use of phase analysis. J Am Coll Cardiol 1989;13:882-90.

7. Cohen TJ, Tucker KJ, Abbott JA, Botvinick EH, Foster E, Schiller $\mathrm{NB}$, et al. Usefulness of adenosine in augmenting ventricular preexcitation for noninvasive localization of accessory pathways. Am J Cardiol 1992;69:1178.

8. Oeff M, Scheinman MM, Abbott JA, Botvinick EH, Griffin JC, Herre JM, et al. Phase image triangulation of accessory pathways in patients undergoing catheter ablation of posteroseptal pathways. PACE 1991;14:1072-80.

9. Rosenqvist M, Isaaz K, Botvinick EH, Dae MW, Cockrell J, Abbott JA, et al. Relative importance of activation sequence compared to atrioventricular synchrony in left ventricular function. Am J Cardiol 1991;67:148-56.

10. Botvinick E, Davis J, Dae M, O'Connell J, Schechtmann N, Abbott J, et al. Localization of ventricular tachycardia exit site and subsequent contraction sequence and functional effects with bedside radionuclide angiography. J Am Coll Cardiol Imaging 2008;1:605-13.

11. O'Connell JW, Schreck C, Moles M, Badwar N, DeMarco T, Olgin $\mathrm{J}$, et al. A unique method by which to quantitate synchrony with equilibrium radionuclide angiography. J Nucl Cardiol 2005; 12:441-50.

12. Cleland JG, Daubert JC, Erdmann E, Freemantle N, Gras D, Kappenberger L, et al. Longer-term effects of cardiac resynchronization therapy on mortality in heart failure. Eur Heart J 2006;27:1928-32.
13. Rivero-Ayerza M, Theuns DA, Garcia-Garcia HM, Boersma E, Simoons M, Jordaens LJ. Effects of cardiac resynchronization therapy on overall mortality and mode of death: A meta-analysis of randomized controlled trials. Eur Heart J 2006;27:2682-8.

14. Pires LA, Abraham WT, Young JB, Johnson KM. MIRACLE and MIRACLE-ICD Investigators. Clinical predictors and timing of New York Heart Association class improvement with cardiac resynchronization therapy in patients with advanced chronic heart failure. Am Heart J 2006;151:837-43.

15. Ypenburg C, Schalij MJ, Bleeker GB, Steenjijk P, Boersma E, Dibbets-Schneider P, et al. Impact of viability and scar tissue on response to cardiac resynchronization therapy in ischaemic heart failure patients. Eur Heart J 2007;28:33-41.

16. Harris A. Delayed development of ventricular ectopic rhythms following experimental coronary occlusion. Circulation 1950; 1:318-24.

17. Kelback H. Technetium-99m labeling of red blood cell: In vitro evaluation of a new approach. J Nucl Med 1986;271:1770-5.

18. Kupersmith J, Krongrad E, Waldo AL. Conduction intervals and conduction velocity in the human cardiac conduction system. Studies during open-heart surgery. Circulation 1973;47:776.

19. Fauchier L, Marie O, Casset-Senon D, Babuty D, Cosnay P, Fauchier JP. Interventricular and intraventricular dyssynchrony in idiopathic dilated cardiomyopathy. A prognosis study with Fourier phase analysis of radionuclide angioscintigraphy. J Am Coll Cardiol 2002;40:2022-30.

20. Kerwin WF, Botvinick EH, O'Connell JW, Merrick SH, DeMarco $\mathrm{T}$, Chatterjee $\mathrm{K}$, et al. Ventricular contraction abnormalities in dilated cardiomyopathy: Effect of biventricular pacing to correct interventricular dyssynchrony. J Am Coll Cardiol 2000;35:1221-7.

21. Badhwar N, O'Connell JW, Green D, Botvinick EH. Equilibrium radionuclide angiogram derived measures of dyssynchrony correlate with clinical outcomes in heart failure patients. J Nucl Med 2008;49:128P (abstr).

22. Burri $H$. The quest for an optimal left ventricular lead position for cardiac resynchronization therapy. Heart Rhythm 2006;3:1293-4.

23. Badhwar N, Viswanathan M, O'Connell JW, DeMarco T, Schreck $\mathrm{C}$, Lee BK, et al. Utility of equilibrium gated radionuclide angiography to guide coronary sinus lead placement in heart failure patients requiring cardiac resynchronization therapy. J Nucl Med 2006;47:1P (abstr).

24. Zaret BL. Cardiac imaging and cardiac resynchronization therapy: Time to get in phase. J Am Coll Cardiol Imaging 2008;1:614-6.

25. Botvinick EH, O'Connell JW, Kadkade PJ, Glickman SL, Dae MW, Cohen TJ, et al. The potential added value of three-dimensional reconstruction and display of SPECT gated blood pool images. J Nucl Cardiol 1998;25:509-14. 